\title{
Exposition des plantations de palmier à huile au risque de la pourriture du cœur dans le bassin de Quevedo, Équateur
}

\author{
Victor Baron $^{1}{ }_{*}$, Sylvain Rafflegeau ${ }^{4}$, Bernard Dubos ${ }^{1}$, Albert Flori ${ }^{1}$, Roberto Burgos ${ }^{2}$ \\ et Claude Louise ${ }^{3}$ \\ ${ }^{1}$ CIRAD, UPR Systèmes de pérennes, 34398 Montpellier, France \\ 2 Asociación Nacional de los Cultivadores de Palma Aceitera (ANCUPA), Quito, Équateur \\ 3 PalmElit, 34980 Montferrier-sur-Lez, France \\ ${ }^{4}$ CIRAD, UMR System, 34060 Montpellier, France
}

\begin{abstract}
Résumé - La maladie de la pourriture du cœur (PC) est la principale menace qui pèse sur la production d'huile de palme en Équateur. Des entretiens individuels menés auprès de 64 chefs d'exploitations élaeicoles du bassin de production de Quevedo ont permis d'évaluer l'exposition des palmeraies à la PC. Les enquêtes ont révélé que seulement $9 \%$ des exploitants adoptent des pratiques préventives et $17 \%$ des pratiques curatives. Seul $27 \%$ des producteurs ont une bonne connaissance de la maladie et des méthodes de lutte recommandées. Une analyse spatialisée des types d'exploitations et des conduites techniques a permis d'identifier les zones les plus sensibles au sein du bassin de production. Le Nord-Ouest du bassin de production est très sensible en raison d'une forte densité de petites exploitations familiales possédant des parcelles anciennes conduites de manière extensive, sans suivi phytosanitaire. À l'opposé, la sensibilité à la maladie est moindre au Sud du bassin de production qui regroupe une majorité d'exploitations managériales avec une meilleure connaissance de la PC, des conduites plus intensives et un suivi phytosanitaire régulier.
\end{abstract}

Mots clés : palmier à huile / Équateur / pourriture du cœur / planteurs indépendants / typologie d'exploitations

\begin{abstract}
Oil palm smallholdings and the risk of bud rot disease in the production area of Quevedo, Ecuador. The bud rot disease is the main threat to the palm oil industry in Ecuador. In the oil palm production area of Quevedo we evaluated smallholders' sensitivity to the risk of bud rot disease, through interviews conducted with 64 oil palm growers. They showed that only $9 \%$ of them implemented preventive measures and $17 \%$ adopted curative measures. Only $27 \%$ of farmers were aware of the disease and control measures. We used a spatialized typology of smallholdings to identify areas that would be the most sensitive to bud rot. The Northwest of the production area was considered as very sensitive because of a high percentage of family farm smallholdings with old plots managed extensively and with poor phytosanitary control. In contrast, the south of the production area was considered less exposed to the bud rot disease as it comprised mostly of holdings well managed and oil palm growers were well aware of the bud rot disease, with a more intensive management and a regular phytosanitary control.
\end{abstract}

Keywords: oil palm / Ecuador / bud rot / smallholders / farm typology

\section{Introduction}

L'huile de palme est aujourd'hui la première source de corps gras au niveau mondial. Cette plante extrêmement productive est cultivée dans de nombreux pays de la zone intertropicale, en Asie, Afrique et Amérique latine. On compte environ 950000 ha de palmier à huile répartis sur le continent américain et l'Équateur est aujourd'hui le septième producteur mondial d'huile de palme, avec une production annuelle de

\footnotetext{
$\overline{\text { *Auteur de correspondance }}$ : victor.baron@cirad.fr
}

510000 tonnes produites sur environ 240000 ha (Oil World, 2015). L'Asociación Nacional de Cultivadores de Palma Aceitera (ANCUPA, 2005) a recensé les exploitations élaeicoles selon un simple critère de surface de palmeraie: les petites palmeraies (moins de 50 ha) représentent environ $40 \%$ des surfaces cultivées en Équateur. Les petits planteurs (smallholders) (Euler et al., 2016) exploitent des palmeraies non industrielles (Rafflegeau, 2007) appelées communément «palmeraies villageoises» (oil palm smallholdings) (Barral et Ruf, 2012). Le terme "petits planteurs» correspond en réalité à une grande diversité de systèmes de production, allant 


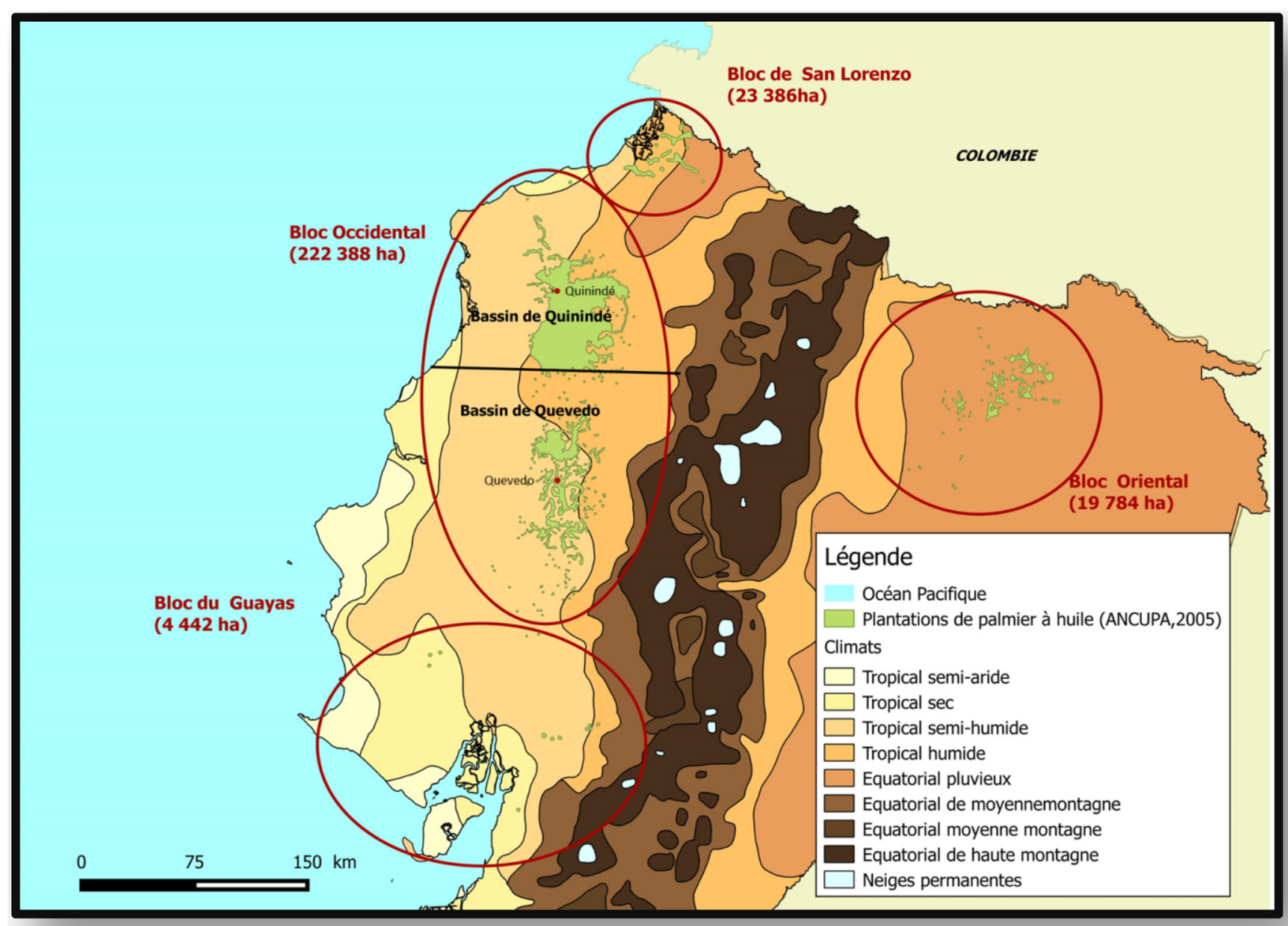

Fig. 1. Répartition des palmeraies en Équateur (données ANCUPA, 2005).

Fig. 1. Palm oil production area in Ecuador (Data ANCUPA, 2005).

de petites exploitations familiales de quelques hectares à de grands projets de plusieurs centaines d'hectares gérés par des investisseurs. Parmi ces exploitations, les différences de conduites techniques et les écarts de rendement peuvent être très importants. Les rendements les plus faibles sont de l'ordre de 3-5 tonnes de régimes par hectare et par an, quand certains « petits planteurs » produisent jusqu'à 25-30 tonnes, c'est-àdire autant que les agro-industries (Winterhalter, 2013).

En Amérique latine, la production d'huile de palme est menacée par la pourriture du cœur (PC), une maladie spécifique au palmier à huile présente uniquement sur ce continent, avec des symptômes très variables (Laing, 2011). Les formes les plus agressives sont présentes en Colombie et en Équateur (Corley et Tinker, 2016). Pour Nieto et al. (1996), il s'agirait d'un complexe regroupant des espèces du genre Fusarium, Thielaviopsis et Phytium. Velez et al. (2008) ont privilégié Phytophtora palmivora comme agent causal, en utilisant des isolats tirés des palmeraies colombiennes proches du bloc de San Lorenzo. En Équateur, Ronquillo-Narváez (2012) ont identifié Fusarium ssp. comme la cause principale de la PC. Quelques auteurs (Laing, 2011; Munevar et al., 2001) ont retenu des facteurs abiotiques comme cause principale de la PC. En l'absence d'un consensus scientifique, dans la suite de cet article, le terme PC désigne un complexe de maladies ayant en commun des pourritures humides des feuilles centrales pouvant entraîner la mort du palmier quand le méristème est atteint (De Franqueville, 2003).

En Équateur, la PC représente la première cause de perte de rendement au niveau national (Naranjo, 2014). Cette maladie affecte différemment chaque zone de production du pays mais touche les agro-industries comme les petits planteurs. Le recensement exhaustif des palmeraies réalisé par l'ANCUPA identifie quatre blocs de plantations (Fig. 1). Le bloc Occidental, le plus vaste avec plus de $80 \%$ des surfaces totales (ANCUPA, 2005), se compose de deux bassins de production autour des agglomérations de Quinindé et de Quevedo. Le bassin de Quevedo est composé uniquement de plantations villageoises, tout comme le bloc Guayas. Le bloc de San Lorenzo est principalement constitué de plantations agroindustrielles. Le bassin de Quinindé et le bloc Oriental associent plantations villageoises et agro-industries.

On distingue en Équateur quatre formes de PC dont trois concernent l'Ouest équatorien (Tab. 1). La maladie est apparue en Équateur en 1976 sur les plaines côtières du bloc Occidental (Louise et al., 2007). Il s'agissait de cas isolés des formes létales comme la pourriture du cœur diffuse (PCD) ou non 
Tableau 1. Épidémiologie et symptomatologie des différentes formes de pourriture du cœur (PC) rencontrées dans l'Ouest équatorien (Louise et al., 2007); données personnelles (Claude Louise).

Table 1. Symptoms and epidemiology of the different forms of bud rot existing in western Ecuador (Louise et al., 2007); personnal data (Claude Louise).

\begin{tabular}{llll}
\hline Forme de la PC & Symptômes/caractéristiques & Incidence/progression de la maladie & Zones touchées \\
\hline Pourriture du cour diffuse & Rémission temporaire & Progression linéaire au départ puis & Équateur: Oriente, Occidente, \\
(PCD) & Feuilles rachitiques & exponentielle & San Lorenzo \\
& Pas de pourriture des régimes & Formation de foyers de contagions & Cas semblables rencontrés dans \\
& & Mortalité élevée & toute l'Amérique latine \\
& & (Brésil, Colombie)
\end{tabular}

Pourriture de flèche $(\mathrm{PF}) \quad$ Pourriture et chute de la flèche Cas diffus, non regroupé en foyer Émission de feuilles courtes Pas de pourriture des régimes

PC de San Lorenzo ou Enfermedad Non Identificada de San Lorenzo (ENI)
Fibres noires et nodules à la base du stipe Pourriture de régimes
Rémission très fréquente, sauf attaque secondaire

Progression foudroyante

Épidémiologie agressive, rémission rare

Plantation ravagée en 2-3 ans

Rémission possible (à confirmer)
Cas isolés partout en Équateur

Similaire à la $\mathrm{PC}$ des

Llanos colombiens

Équateur: San Lorenzo, Nord de Quinindé (Viche)

Colombie: Tumaco létales comme la pourriture de flèche (PF). En 2004-2005, une forme létale de PC est apparue au Sud de la Colombie dans la région de Tumaco. En 2007, elle a touché les plantations de San Lorenzo où elle a été appelée PC «San Lorenzo» ou Enfermedad Non Identificada (ENI) (Bernal, 2014). Sa progression fulgurante a dévasté 15000 des 20000 ha de San Lorenzo entre 2009 et 2014 (Naranjo, 2014). La maladie est ensuite apparue en 2010 dans le bassin de production de Quinindé, à $200 \mathrm{~km}$ au Sud de San Lorenzo, qui compte de très nombreux petits planteurs. En 2014, un recensement phytosanitaire a été effectué par l'ANCUPA dans sept cantons au Nord du bassin de Quinindé (Fig. 2). Toutes formes de PC confondues, sur 13267 ha recensés, 8313 ha étaient touchés et 3013 ha complètement dévastés. En raison de la progression très rapide de l'ENI, certains agriculteurs avaient cessé l'exploitation, abandonnant leurs parcelles dans l'état (Naranjo, 2014).

Les petits planteurs touchés par la PC disposent de peu de moyens de prévention et de lutte pour répondre à la maladie, à la différence des agro-industries qui ont opté pour l'utilisation de l'hybride entre l'espèce sud-américaine Elaeis oleifera et l'espèce africaine Elaeis guineensis (hybride OxG). Le matériel végétal $\mathrm{OxG}$ s'est révélé très résistant à la forme PCD dans le bloc Oriental (Louise et al., 2007) et plus récemment pour l'ENI à San Lorenzo. Cette méthode de lutte est de loin la plus efficace et a été largement adoptée par les agro-industries, mais présente l'inconvénient d'exiger une pollinisation manuelle. Au sein de l'espèce Elaeis guineensis, qui n'exige pas de pollinisation manuelle (sauf à l'entrée en production si les conditions sont très favorables), plusieurs catégories commerciales regroupées sous le nom de «El Dorado » présentent une résistance intermédiaire.

Plusieurs macro-acteurs privés et institutionnels jouent un rôle important dans la formation et le conseil technique des producteurs, notamment l'ANCUPA (association regroupant $75 \%$ des élaeiculteurs du pays); Agrocalidad (autorité nationale chargée des questions phytosanitaires) ; les fournisseurs de matériel végétal sélectionné; et les huileries, qui achètent les régimes et peuvent fournir des intrants aux producteurs. Les méthodes de lutte préventives, recomman- dées en Équateur par l'ANCUPA et Agrocalidad, forment un ensemble de «bonnes pratiques agricoles »: origine contrôlée du matériel végétal, drainage des sols, présence d'une plante de couverture, nutrition minérale équilibrée et lutte contre Rhynchophorus palmarum, un coléoptère dont les attaques aggravent les dégâts dus à la PC. Les méthodes de lutte curatives consistent à détecter précocement les symptômes de $\mathrm{PC}$ et à pratiquer une ablation des tissus nécrosés (Martínez et al., 2008), une technique surtout efficace sur la forme PF et pour les jeunes palmiers. Les palmiers âgés où l'on détecte souvent des cas déjà avancés du fait de la hauteur qui rend difficile l'observation des feuilles, doivent être abattus pour éradiquer le foyer (Bernal et al., 2013).

Compte tenu du caractère létal de certaines formes de PC et de l'ampleur des dégâts constatés récemment, il est nécessaire de comprendre les répercussions que la maladie pourrait avoir dans les zones encore indemnes du bassin de Quevedo. Quelle est la capacité des exploitants à anticiper ou à prévenir la maladie? Comment protéger cette culture au cours des prochaines années? En l'absence d'un consensus scientifique sur l'épidémiologie et la symptomatologie de la maladie, nous avons choisi d'aborder la question des risques phytosanitaires en nous intéressant aux agriculteurs, à leurs exploitations et systèmes de production. Dans un bassin composé essentiellement de petits planteurs, nous cherchons à identifier, avant l'arrivée d'une maladie dévastatrice, les exploitations les plus exposées d'un point de vue phytosanitaire pour mieux cibler les messages de prévention et les interventions techniques.

\section{Méthodologie}

\subsection{Collecte des données}

Soixante-quatre chefs d'exploitations ont été tirés au hasard parmi l'ensemble des 1753 petits planteurs du bassin de production de Quevedo, à partir des listes fournies par les cinq huileries couvrant le bassin. Il s'agit d'un échantillonnage aléatoire stratifié avec allocation proportionnelle, où le nombre de planteurs sélectionnés par huilerie est proportionnel à 


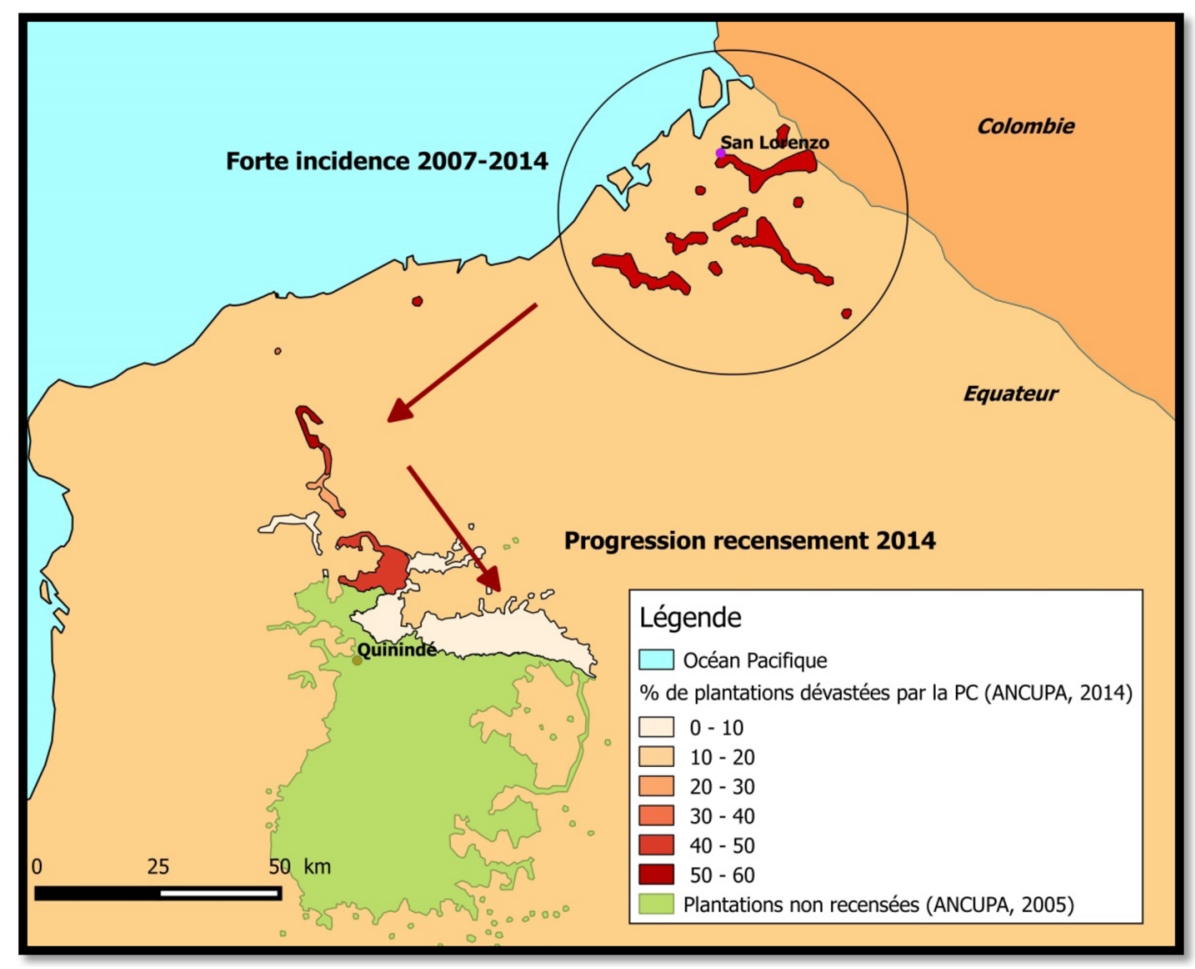

Fig. 2. Progression de la pourriture du cœur (PC) dans 1'Ouest équatorien entre 2007 et 2014 (données ANCUPA, 2005, Naranjo 2014). Fig. 2. Territorial progression of the bud rot disease in western Ecuador 2007-2014 (data ANCUPA, 2005, Naranjo 2014).

Tableau 2. Répartition de l'échantillon des planteurs pour chaque strate correspondant aux huileries.

Table 2. Stratification of the sample of planters, per palm oil mill.

\begin{tabular}{lrcc}
\hline Huilerie & \multicolumn{2}{c}{ Nombre de fournisseurs } & Échantillon \\
\hline Agroparaiso & 600 & $34 \%$ & 21 \\
Oleorios & 172 & $10 \%$ & 6 \\
Palmisa & 82 & $5 \%$ & 3 \\
Quevepalma & 370 & $21 \%$ & 14 \\
Rio Manso & 529 & $30 \%$ & 20 \\
Total & 1753 & & 64 \\
\hline
\end{tabular}

l'importance de celle-ci dans la population totale des planteurs du bassin (Tab. 2).

Les entretiens avec les agriculteurs furent réalisés individuellement, au sein des exploitations avec souvent une visite des parcelles. L'entretien était divisé en trois parties. La première partie visait à comprendre la trajectoire personnelle de l'agriculteur et sa situation économique, la taille et la structure de son exploitation, les systèmes de cultures présents, et le rôle des parcelles de palmiers dans l'exploitation. La seconde portait sur les systèmes de culture du palmier à huile. Chaque parcelle de l'exploitation est caractérisée par sa taille et l'âge des palmiers, puis par neuf variables agronomiques: matériel végétal, légumineuse de couverture, amendement organique, cultures associées, type d'engrais utilisé, quantité d'engrais appliqué, fractionnement des engrais, fertilisation en phase juvénile. Ces pratiques culturales sont susceptibles d'avoir un impact sur l'état du peuplement végétal en plantations villageoises (Rafflegeau et al., 2010) et furent retenues comme déterminantes dans le diagnostic agronomique régional réalisé en 2013 dans la région de Quinindé (Rafflegeau et al., 2015). La dernière partie a permis d'identifier le niveau d'incidence de la PC, le niveau de connaissance de la maladie et les éventuelles pratiques préventives et curatives mises en place dans l'exploitation.

\subsection{Analyse des données collectées}

L'analyse des systèmes d'exploitation est basée sur la typologie définie par Marzin et al. (2014), qui distinguent trois types d'agriculture en fonction de l'organisation du travail: familial, patronal et managérial. Le critère de la diversification $\mathrm{du}$ système d'activité y a été ajouté, car les activités rémunératrices non agricoles influent sur les stratégies de conduites techniques des palmeraies en modifiant les flux de trésorerie et l'allocation du temps de travail (Baron, 2014). Les petits planteurs sont répartis en cinq classes définies à partir de deux critères principaux : l'organisation du travail au sein de l'exploitation, ainsi que la nature principale ou secondaire des activités agricoles dans leur système d'activité. Les petits planteurs ont également été répartis en trois classes selon leur connaissance de la symptomatologie de la PC et des méthodes de luttes existantes.

À l'échelle de la parcelle, la typologie des conduites techniques a été construite en réalisant une analyse des correspondances multiples (ACM) sur les neuf variables agronomiques étudiées, sans prendre en compte les types d'exploitation dans lesquels ces parcelles se trouvent. Les résultats de l'ACM traités par une classification ascendante 
Tableau 3. Répartition de l'échantillon en fonction du type d'exploitation agricole. Les différences de surface moyenne ont été testées par ANOVA et sont hautement significatives $(\mathrm{ddl}=4, p$ value $<0,0001)$, les moyennes qui ne présentent pas de lettre commune sont significativement différentes au seuil de $5 \%$ (test de Tukey).

Table 3. Description of the different types of farming systems within our sample. Analysis of variance showed that the average acreage were significantly different between farming system (ddl=4, $\mathrm{p}$ value $<0.0001)$, means with no common letter are significantly different (Tukey test).

\begin{tabular}{lccccc}
\hline Type d'exploitation & Effectifs & SAU moyenne (ha) & Effectifs (\%) & Total des surfaces palmiers & Surfaces totales (\%) \\
\hline Familiale & 15 & $13^{\mathrm{a}}$ & 23 & 126 & 4 \\
Patronale agricole & 15 & $34^{\mathrm{a}}$ & 23 & 352 & 12 \\
Patronale investisseur & 16 & $62^{\mathrm{a}, \mathrm{b}}$ & 25 & 664 & 23 \\
Managériale agricole & 14 & $154^{\mathrm{b}}$ & 22 & 1132 & 38 \\
Managériale investisseur & 4 & $307^{\mathrm{c}}$ & 6 & 673 & 23 \\
Total général & 64 & & & 2947 & \\
\hline
\end{tabular}

hiérarchique $(\mathrm{CAH})$ ont abouti à une répartition des 185 parcelles présentes chez les 64 planteurs étudiés en quatre classes de conduite technique. L'âge des parcelles a été ajouté comme co-variable pour chaque classe.

La distribution des types de conduites techniques en fonction des types d'exploitations a été étudiée en croisant ces variables dans des tableaux de contingences. Les exploitations furent repérées dans l'espace par leurs coordonnées géographiques et le logiciel libre Quantum Geographic Information System (QGIS) a été utilisé pour élaborer un zonage du bassin de production. La caractérisation de chacune de ces zones a été affinée par des entretiens auprès de macro-acteurs de la filière (huileries, ANCUPA, fournisseurs de matériel végétal) permettant de valider l'histoire du développement élaeicole en dessinant les différentes zones d'implantation successives de la culture.

\section{Résultats}

\subsection{Typologie des exploitations et des conduites techniques}

Le verger du bassin de Quevedo est globalement jeune et issu d'une dynamique récente de conversion des terres agricoles, les palmeraies étant majoritairement implantées sur des précédents maïs et soja (39\% des surfaces), cacao $(16 \%)$ et prairies $(14 \%)$. Seulement $7 \%$ des surfaces sont en deuxième cycle de culture du palmier et $60 \%$ des surfaces en production ont moins de 10 ans. Les palmeraies juvéniles (moins de 3 ans) représentent $26 \%$ des surfaces.

La typologie des exploitations a abouti à une répartition des élaeiculteurs en cinq classes présentées dans le Tableau 3. Les exploitations familiales sont de taille réduite, où les propriétaires vivent à proximité des parcelles qu'ils exploitent en utilisant la main d'œuvre familiale et parfois des journaliers pour la récolte. Le type patronal agricole regroupe également des petites exploitations où les propriétaires vivent à proximité des champs, mais où les travaux sont réalisés en majorité par des employés. Les exploitations managériales agricoles sont de grandes propriétés n'employant que des salariés, dans lesquelles les propriétaires ont généralement suivi une formation agricole et disposent de moyens techniques et financiers importants. Il s'agit souvent d'exploitations bananières ou de grandes cultures qui convertissent pro- gressivement leurs terres à l'élaeiculture. Les exploitations de type « investisseur» (patronales ou managériales) correspondent à des situations où l'agriculture n'est pas l'activité principale du propriétaire, qui ne réside pas à proximité de la plantation. Pour les exploitations patronales-investisseurs, il s'agit de personnes ayant hérité ou possédant des palmeraies villageoises comme revenu complémentaire, gérées à distance en minimisant le temps passé sur l'exploitation. Les exploitations managériales-investisseurs correspondent à des élites économiques locales qui diversifient leurs investissements et qui délèguent toute la gestion à des chefs d'exploitation présents sur place.

L'analyse statistique classe les parcelles étudiées en quatre classes de conduites techniques selon un gradient d'intensification du travail et des intrants (Tab. 4). La classe «matériel végétal tout venant » (MVTV) utilise un matériel végétal non certifié Tenera issu de la fécondation libre de palmiers sélectionnés, qui ne produit que $40 \%$ de l'huile produite par le matériel sélectionné dans les mêmes conditions (Cochard et al., 2001). Ces parcelles sont souvent âgées, avec une faible densité de plantation (moins de 120 palmiers par hectare) et associées à de l'élevage. L'application des pratiques recommandées (matériel sélectionné, plante de couverture, fertilisation minérale et recyclage des sous-produits organiques) améliore la productivité, avec un gain de l'ordre de 6 à 10 tonnes par hectare (Winterhalter, 2013). La classe « infrarecommandation » (INFRA) concerne des parcelles plantées avec du matériel sélectionné recevant une faible dose de fumure minérale et plantées avec une densité faible permettant une association avec le maïs au jeune âge. La classe « intermédiaire » (INTER) regroupe un ensemble de pratiques culturales plus variables caractérisées par des cultures associées fréquentes et des doses d'intrants inférieures à la classe «intensif» (INTEN). La classe INTEN représente le niveau d'intensification le plus élevé avec l'application des recommandations, avec parfois des cultures associées au jeune âge.

Le Tableau 5 croise les types de conduites techniques et les types d'exploitations. Il montre la cohabitation de plusieurs types de conduites au sein d'une même exploitation, les planteurs faisant évoluer leurs pratiques au fil des implantations successives des parcelles. Les exploitations familiales ne présentent que des conduites techniques de type INFRA ou MVTV. Le reste des parcelles MVTV et INFRA sont présentes 
V. Baron et al. : Cah. Agric. 2017, 26, 55002
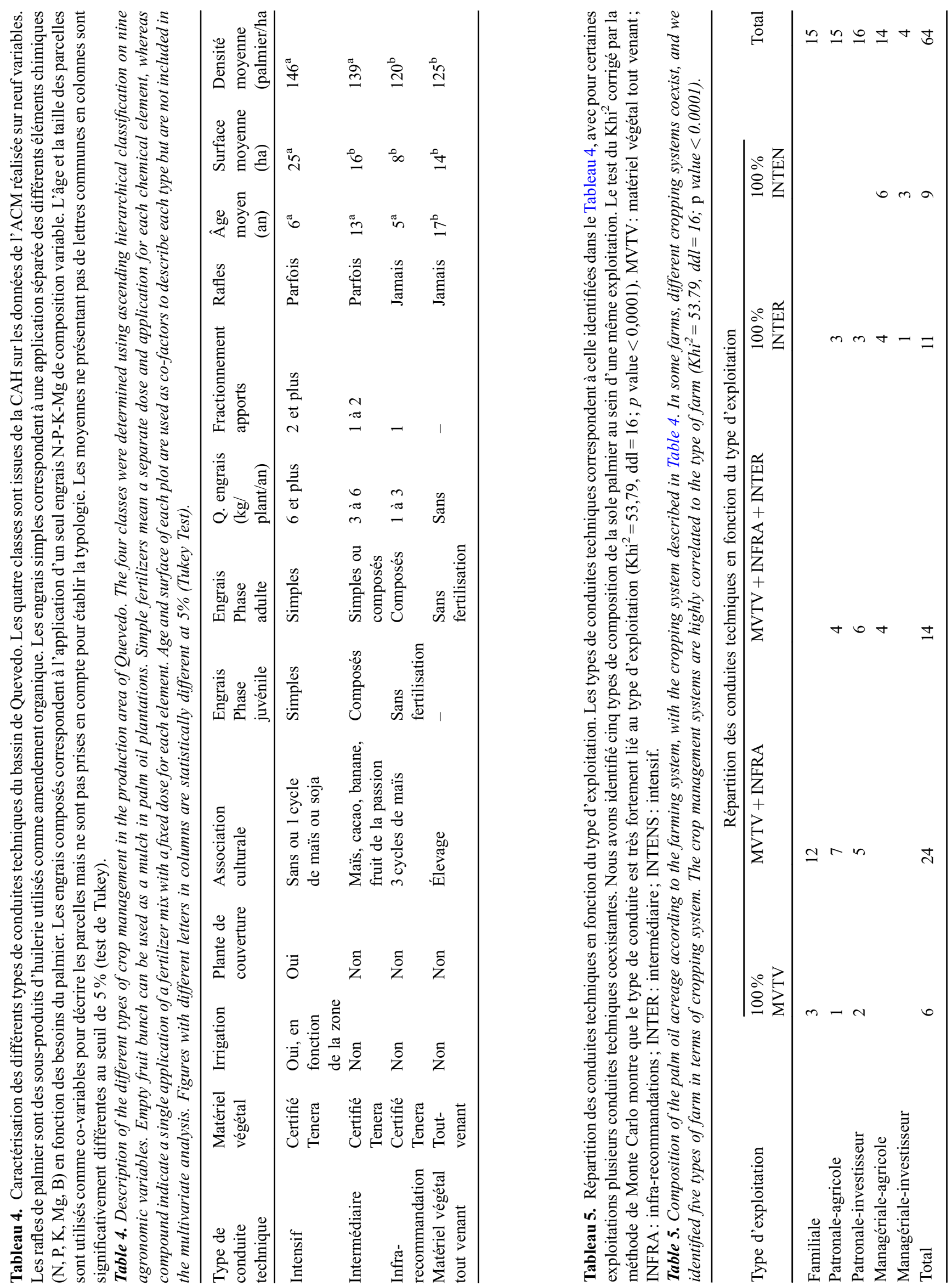
en majorité dans les exploitations patronales. Les exploitations managériales ont des conduites plus intensives de type INTER ou INTEN.

\subsection{Stratégies des élaeiculteurs vis-à-vis du risque PC}

\subsubsection{Incidence et traitement de la maladie}

Les informations collectées auprès des petits planteurs indiquent que l'ENI, forme la plus agressive de la maladie, n'était pas présente dans le bassin de Quevedo en 2014. Cependant, $65 \%$ (intervalle de confiance à $95 \%[\mathrm{IC}]=54$ $77 \%$ ) des planteurs ont déjà été confrontés à une autre forme de PC; il s'agissait probablement de PF ou de cas isolés de PCD. Les cas reportés sont dispersés et aucun foyer important n'a été recensé lors de nos enquêtes.

Les données du Tableau 6 montrent que lorsque aucun cas de PC n'a été détecté dans leur exploitation, les planteurs n'appliquent aucune mesure préventive. Dans le bassin de production, seulement $6 \%(\mathrm{IC}=0,2-16 \%)$ des exploitations mettent en place des pratiques préventives et $17 \%(\mathrm{IC}=8-$ $26 \%$ mettent en place des pratiques curatives. L'adoption de matériel végétal $\mathrm{OxG}$ résistant est anecdotique.

\subsubsection{Niveau de connaissance des agriculteurs}

Le Tableau 7 montre que seulement 26\% (IC = 15-37\%) des planteurs ont une bonne connaissance de la maladie et de l'existence de matériel tolérant. Ces savoirs ont surtout été acquis dans les exploitations managériales alors que les petits planteurs des exploitations familiales sont significativement moins bien informés sur la maladie et sur les méthodes de luttes disponibles. L'enquête a aussi révélé que très peu de planteurs $(13 \%, \mathrm{IC}=6-24 \%)$ ont une bonne connaissance du matériel OxG. Pour certains la pollinisation manuelle est un frein à son adoption. L'existence et les caractéristiques du matériel El Dorado sont méconnues par 97\% (IC=88-99\%) des planteurs.

\subsection{Dynamiques de plantations}

Le positionnement des plantations dans l'espace et les enquêtes complémentaires menées auprès des macro-acteurs $\mathrm{du}$ secteur ont permis de reconstituer une chronologie d'extension des plantations à partir des années 1980 jusqu'à aujourd'hui. Quatre zones d'extension de la culture du palmier ont été identifiées (Fig. 3):

- zone de Buena Fe : la culture du palmier à huile a démarré dans le bassin de Quevedo à la fin des années 1980, quand un petit groupe d'agriculteurs possédant de grandes exploitations (50 à 200 ha) a commencé à planter puis à investir dans de mini-huileries. C'est dans cette zone que se situent les anciennes palmeraies de la région, dans des exploitations managériales-agricoles. Se sont ensuite ajoutées des exploitations patronales-agricoles et patronales-investisseurs. Il existe encore de nos jours des parcelles âgées (plus de 20 ans) dans cette zone où la dynamique de plantations est actuellement faible;

- zone de Manga del Cura: la présence des huileries de Buena Fe a suscité l'apparition de petites exploitations
Tableau 6. Pratiques phytosanitaires en fonction de l'incidence de la maladie (en nombre de planteurs). Incidence nulle: aucun cas de pourriture du cœur (PC) détecté dans l'exploitation. Incidence faible moyenne : quelques cas de PC ont été détectés dans l'exploitation mais l'incidence de la maladie n'affecte pas significativement le rendement. Forte: le nombre de cas de $\mathrm{PC}$ affecte la production à l'échelle de l'exploitation.

Table 6. Phytosanitary treatments and actual impact of the disease. No incidence: no case of bud rot was observed. Medium incidence: a few cases have been detected but it does not affect the overall production. Strong incidence: many cases have been observed and the overall production is affected by the bud rot.

\begin{tabular}{|c|c|c|c|c|c|}
\hline $\begin{array}{l}\text { Incidence } \\
\mathrm{PC}\end{array}$ & $\begin{array}{l}\text { Aucun } \\
\text { traitement }\end{array}$ & $\begin{array}{l}\text { Traitement } \\
\text { curatif }\end{array}$ & $\begin{array}{l}\text { Traitement } \\
\text { préventif }\end{array}$ & $\begin{array}{l}\text { Matériel } \\
\text { végétal } \\
\text { résistant }\end{array}$ & \\
\hline Nulle & 22 & & & & 22 \\
\hline $\begin{array}{l}\text { Faible à moyenne } \\
\text { Forte }\end{array}$ & 26 & 11 & 4 & 1 & 42 \\
\hline Total & 48 & 11 & 4 & 1 & 64 \\
\hline
\end{tabular}

Tableau 7. Connaissance de la pourriture du cœur (PC) au sein des exploitations. Le niveau de connaissance est très fortement dépendant du type d'exploitation (test du $\mathrm{Khi}^{2}$ corrigé par la méthode de MonteCarlo: $\mathrm{Khi}^{2}=23285$, ddl $=4 ; p$ value $=0,003$ ).

Table 7. Knowledge about the bud rot disease. The knowledge is highly correlated to the type of farm $\left(K h i^{2}=23285, d d l=4 ; \mathrm{p}\right.$ value $=0.003$ ).

\begin{tabular}{lrrrl}
\hline Type exploitation & Faible & Moyenne & Bonne & $\begin{array}{l}\text { Total } \\
\text { général }\end{array}$ \\
\hline Familiale & 10 & 4 & 1 & 15 \\
Patronale & 7 & 7 & 1 & 15 \\
Patronale diversifiée & 5 & 7 & 4 & 16 \\
Managériale agricole & 1 & 5 & 8 & 14 \\
Managériale grand investisseur & & 1 & 3 & 4 \\
Total général & 23 & 24 & 17 & 64
\end{tabular}

Connaissance faible : a déjà rencontré des cas de PC au champ, mais ne connaît ni les pratiques de prévention ni celles de traitement curatif; connaissance moyenne: connaît les pratiques de traitement curatif et certaines pratiques préventives. $\mathrm{Ne}$ connaît pas ou faiblement le matériel résistant $\mathrm{OxG}$; connaissance bonne : connaît les pratiques de prévention et de traitement curatif. Est capable de détecter les symptômes précoces de la maladie. Connaît l'existence et les caractéristiques du matériel OxG résistant.

familiales dans la zone enclavée de Manga Del Cura dans les années 1990. L'implantation de la culture s'est faite au départ avec des conduites de type MVTV. On y retrouve aujourd'hui des parcelles récentes de type INFRA et des parcelles anciennes de type MVTV. Plus récemment sont apparues des exploitations patronales-investisseurs, avec des conduites INFRA ou INTER;

- zone du Canton de Quevedo: au début des années 2000, l'extension des surfaces cultivées s'est faite autour de Quevedo, notamment grâce à deux nouvelles huileries 


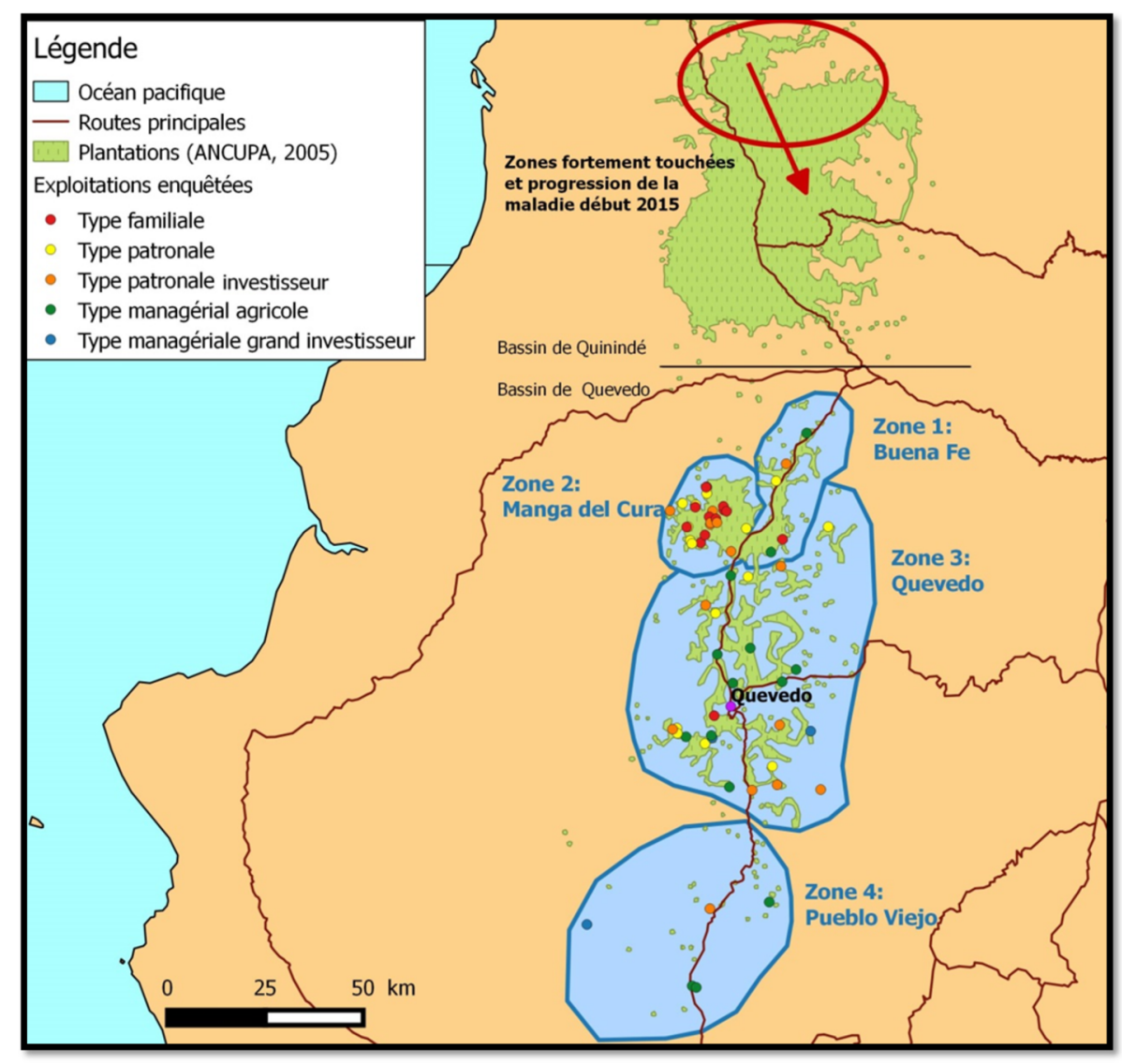

Fig. 3. Zonage du bassin de production (données ANCUPA, 2005 et données de l'étude).

Fig. 3. Delimitation of zones within the production areas (data ANCUPA, 2005 and results of the study).

proches de la ville. Les types d'exploitation dans cette zone sont très variés, avec une majorité d'exploitations patronales-agricoles, patronales-investisseurs et managériales-agricoles. Toutes les palmeraies récentes sont sélectionnées. On remarque l'apparition d'exploitations de type "grand investisseur», avec des plantations de grande taille ( $>200 \mathrm{ha}$ ). Ces projets correspondent parfois à la conversion de grandes bananeraies, avec des conduites techniques très intensives en capital et en main d'œuvre;

- zone de Pueblo Viejo: depuis quelques années, une forte dynamique de plantation est apparue au Sud du bassin de production avec des projets de grande ampleur. Les parcelles sont conduites de manière très homogène en adoptant les recommandations techniques vulgarisées. Les propriétaires ont une bonne connaissance technique ou emploient des ingénieurs agronomes comme chef d'exploitation.

\section{Discussion}

De nombreuses études phytopathologiques visent à évaluer les liens entre conditions environnementales, pratiques agricoles, incidences d'une ou plusieurs maladies et performances de la culture (Savary et al., 2006). Ces méthodes dites de recensement (field survey sampling) se concentrent en général sur des données physiques, physiologiques et biologiques collectées au sein d'un échantillon raisonné de parcelles. L'analyse de ces données permet d'effectuer des typologies d'agro-systèmes en fonction de leur sensibilité à une maladie (Allinne et al., 2016; Avelino et al., 2006) ou d'aboutir à la modélisation des pertes de rendements (Madden et Nutter, 1995). Cette étude présente une approche plus agroéconomique, basée sur une caractérisation spatio-temporelle des systèmes de production et s'appuyant sur les connaissances déjà existantes de la maladie. L'intérêt de cette méthode, complémentaire au type d'étude cité ci-dessus, est de pouvoir orienter les campagnes de lutte préventive et curative en s'appuyant sur des considérations socio-économiques. Elle permet de comprendre les structures du bassin de production, d'identifier des types d'exploitations vulnérables et de proposer des modes d'intervention différenciés de la part des macro-acteurs, le tout avant que les exploitations soient touchées par une épidémie.

\subsection{Effets des conduites techniques sur l'incidence et la propagation de la PC}

L'évaluation de l'incidence de la PC, réalisée aux dires d'acteurs, suggère que la pression de la maladie est encore très faible sur l'ensemble du bassin de Quevedo et que la forme létale foudroyante n'y est pas présente. Le niveau d'incidence de la maladie est basé ici sur la perception de l'agriculteur. 
Les exploitations de type familiales possèdent le plus de parcelles peu productives plantées avec du matériel tout venant, conduites de manières extensives avec peu de suivi et de traitements phytosanitaires (type MVTV). Ces parcelles présentent des risques plus grands de contamination par la PC en l'absence de pratiques préventives et de traitement des cas existants. La présence permanente de bétail dans ce type de système (type MVTV) peut aussi en limiter le renouvellement, le chef d'exploitation ayant l'option de ne plus exploiter ses palmiers mais de conserver les parcelles en l'état pour le pâturage. On constate également dans les exploitations familiales et patronales-investisseurs une conduite technique correspondant à une stratégie de minimisation des coûts de production. En cas d'apparition de la PC, ces parcelles pourraient constituer des foyers de contamination en l'absence de suivi phytosanitaire et de traitement curatif.

Les exploitations patronales-agricoles et patronales-investisseurs présentent des pratiques culturales variables pour les différentes parcelles d'une même exploitation (MVTV+ INFRA + INTER). La présence de parcelles exploitées de manière extensive peut être une source de contamination pour l'ensemble de l'exploitation. Cependant, les exploitants ont en général une meilleure connaissance technique et une plus grande capacité de financement que ceux des exploitations familiales, ce qui permet d'espérer une meilleure réponse à la PC.

Les exploitations managériales-agricoles ou managérialesinvestisseurs ont des conduites techniques plus intensives en main d'œuvre et en intrant (type INTEN), avec une logique de maximisation des rendements et une gestion proche de celle des agro-industries. Dans ces exploitations, le suivi phytosanitaire est plus rigoureux et l'emploi de traitements pour la protection des cultures est courant. Les conduites techniques correspondent aux recommandations de l'ANCUPA en termes de pratiques préventives. Ce sont donc les exploitations qui présentent les risques de contamination et de propagation les plus faibles.

\subsection{Quelles sont les principales zones à risques?}

L'étude de l'histoire du développement élaeicole dans le bassin de production a abouti à un zonage qui permet d'examiner la sensibilité de chacune des quatre zones selon deux risques: la baisse des rendements et la hausse de la mortalité dans les parcelles et la propagation de la PC entre les parcelles en l'absence de lutte phytosanitaire appropriée. L'âge moyen des plantations dans une zone est aussi un critère important, car il est plus difficile de détecter et de traiter la maladie sur des palmiers âgés:

- les exploitations familiales et patronales de la zone de Manga Del Cura sont les plus exposées aux risques de mortalité et de propagation de la PC. D'une part, elles sont proches des foyers du bassin de Quinindé et la proximité entre les exploitations favorise la contamination de proche en proche. D'autre part, la connaissance de la PC y est faible et les parcelles âgées conduites sans traitement phytosanitaire (type MVTV et INFRA) sont fréquentes et souvent associées à l'élevage;

- la zone de Buena $\mathrm{Fe}$ présente un niveau de risque intermédiaire. Les plantations y sont assez âgées et également proches du bassin de Quinindé. Les chefs d'exploitations y ont une bonne connaissance technique et le niveau d'intensification est plus important que dans la zone de Manga del Cura. Il existe un risque de contamination des parcelles âgées sans suivi phytosanitaire (type INFRA ou INTER);

- la zone de Quevedo est une zone de risque intermédiaire car les types d'exploitations et de conduites y sont variés. Les plantations sont en moyenne assez jeunes avec très peu de plantations de type MVTV;

- le risque est le plus faible dans la zone de Pueblo Viejo, car les palmeraies sont éloignées les unes des autres et très récentes (moins de 8 ans), ce qui facilite la prévention et le traitement de la maladie. Les conduites intensives (type INTER) laissent présager de l'application des méthodes de lutte préventive et curative en cas d'épidémie de PC.

\subsection{Quelles interventions pour les macro-acteurs?}

Il ressort de cette étude une nécessité pour l'ensemble des acteurs de la filière (Agrocalidad, ANCUPA, huileries, pépiniéristes, semenciers) d'adapter le conseil technique à chaque zone et à chaque type d'exploitation pour minimiser le risque de PC. Dans la zone de Manga del Cura, qui regroupe la majorité des exploitations familiales et patronales, la priorité doit être donnée à la formation des producteurs et à un suivi phytosanitaire régulier de la part des macro-acteurs. Ces agriculteurs représentent un faible pourcentage des surfaces cultivées mais doivent néanmoins bénéficier d'un appui technique important. Dans le cas de parcelles associées à l'élevage, les conseillers et techniciens doivent veiller à l'élimination des palmiers malades. Dans les exploitations patronales regroupant différents types de parcelles (MVTV + INFRA + INTER), la replantation des parcelles MVTV est un enjeu important pour améliorer la productivité et éviter les foyers de contamination. Parmi les types de matériel végétal qui présentent des caractères de résistance aux formes de $\mathrm{PC}$, le matériel guineensis résistant paraît plus adapté aux exploitations familiales et patronales car il ne nécessite pas de pollinisation manuelle. Dans les zones de Quevedo et la zone de Buena $\mathrm{Fe}$, les types d'exploitations élaeicoles sont plus variables et les méthodes de lutte doivent être adaptées au profil de chaque planteur. Le renouvellement des dernières plantations MVTV présentes dans la zone de Buena Fe est également une priorité.

Les exploitations managériales des zones de Quevedo ou Pueblo Viejo constituent une opportunité pour tester l'introduction du matériel $\mathrm{OxG}$ en palmeraies villageoises. Pour l'instant, la PC a touché essentiellement des zones d'agroindustries qui ont eu la capacité de replanter avec du matériel OxG après des épisodes de PC (Dubos et al., 2013; Louise et al., 2007). Or les exploitations managériales de ces deux zones semblent les plus à mêmes de planter du matériel $\mathrm{OxG}$ car leurs conduites techniques intensives sont proches de celles des agro-industries. Des expériences réussies de conduite de palmeraies OxG par des petits planteurs permettraient de prévenir le risque $\mathrm{PC}$ en amont et de replanter rapidement les palmeraies villageoises en cas de dégâts causés par une forme virulente de la maladie. 


\section{Conclusion}

Les élaeiculteurs du bassin de Quevedo sont pour l'instant uniquement confrontés à des formes de PC ayant peu ou pas d'incidence majeure sur la productivité de leurs plantations. Malgré de nombreuses campagnes d'information à travers le pays, les planteurs ne prennent pas assez de mesures pour anticiper les risques liés à une épidémie de PC. Les types de conduites techniques dans le bassin de production sont très variables et dépendent du type d'exploitations. Bien qu'il existe des exploitations familiales ou patronales faisant évoluer leurs pratiques vers une plus grande intensification, on peut dire que ces types d'exploitation présentent plus de parcelles à risque (incidence et propagation), et ont des conduites techniques actuellement peu compatibles avec la plantation de matériel hybride $\mathrm{OxG}$ résistant à la $\mathrm{PC}$. La zone de Manga del Cura est une zone de production importante avec de nombreuses petites exploitations familiales qui pourraient être gravement touchées dans le cas d'une épidémie. La priorité des macro-acteurs consiste à promouvoir le suivi phytosanitaire des parcelles et la plantation de matériel résistant: guineensis ou $\mathrm{OxG}$ si le planteur est susceptible de maîtriser la pollinisation manuelle. Les zones de Quevedo et surtout de Pueblo Viejo semblent être moins exposées. La présence de grandes exploitations intensives dans ces zones constitue une opportunité pour développer l'utilisation de matériel $\mathrm{OxG}$ par certains petits planteurs.

Remerciements. Les auteurs remercient chaleureusement toute l'équipe de PalmElit, en particulier Hubert de Franqueville qui nous a quitté trop tôt. Nous remercions également nos partenaires en Équateur sans qui cette étude n'aurait pas été possible: l'ANCUPA, DANEC, Murrin et l'université de Quevedo.

\section{Références}

Allinne C, Savary S, Avelino J. 2016. Delicate balance between pest and disease injuries, yield performance, and other ecosystem services in the complex coffee-based systems of Costa Rica. Agriculture, Ecosystems \& Environment 222: 1-12. DOI: 10.1016/ j.agee.2016.02.001.

ANCUPA. 2005. Inventario de plantaciones de palma aceitera en el Ecuador. Quito: ANCUPA, 28 p.

Avelino J, Zelaya H, Merlo A, Pineda A, Ordoñez M, Savary S. 2006. The intensity of a coffee rust epidemic is dependent on production situations. Ecological Modelling 197(3-4): 431-447. DOI: 10.1016/j.ecolmodel.2006.03.013.

Baron V. 2014. Dynamiques de plantation et conduite technique du palmier à huile (Elaeis sp.) dans le bassin de production de Los Rios et du Guayas, Équateur. Cergy-Pontoise: ISTOM, 78 p. Mémoire de fin d'études. http://agritrop.cirad.fr/575896/1/docu ment_575896.pdf.

Barral S, Ruf F. 2012. Plantations industrielles ou familiales? Regards croisés sur la production d'huile de palme et de cacao en Indonésie et au Ghana. Autrepart 62(3): 75.

Bernal G. 2014. Breve historia de la pudrición del cogollo en Ecuador. Revista Institucional Palma 25: 20-23.

Bernal G, Ronquillo M, Vega V, Bravo V, Paredes E. 2013. Guía de Campo sobre la Pudricion de Cogollo (PC). Quito: ANCUPA, $26 \mathrm{p}$.
Cochard B, Adon B, Kouame-Jouame R, Durand-Gasselin T, Amblard P. 2001. Intérêts des semences commerciales améliorées de palmier à huile (Elaeis guineensis Jacqu.). Oléagineux Corps Gras Lipides 8: 654-658. DOI: 10.1017/S0014479703001315.

Corley RHV, Tinker PHB. 2016. Diseases of the oil palm. In: The Oil Palm, 5th edition. Chichester: Wiley Blackwell, pp. 399-437.

De Franqueville H. 2003. Oil palm bud rot in Latin America. Experimental Agriculture 39: 225-240. DOI: 10.3917/autr.062.0075.

Dubos B, Gallardo C, Zambrano J. 2013. Comportamiento nutricional de los híbridos interespecificos Elaeis oleifera $\mathrm{x}$ Elaeis guineensis $(\mathrm{OxG})$ en el oriente ecuatoriano y colombiano. Palmas 34: 337-344.

Euler M, Schwarze S, Siregar H, Qaim M. 2016. Oil palm expansion among smallholder farmers in Sumatra, Indonesia. Journal of Agricultural Economics 67(3): 658-676. DOI: 10.1111/ 1477-9552.12163.

Laing D. 2011. La deficiencia transitoria de calcio como la causa primordial de la pudrición de cogollo en la palma de aceite. Informaciones agronómicas del IPNI 3: 26-52.

Louise C, Amblard P, De Franqueville H, Benavides D, Gallardo C. 2007. Investigaciones dirigidas por el Cirad sobre las enfermedades del complejo pudrición del cogollo de la palma aceitera en Latinoamérica. Palmas 28: 345-362.

Madden LV, Nutter FW. 1995. Modeling crop losses at the field scale. Canadian Journal of Plant Pathology 17(2): 124-137. DOI: 10.1080/07060669509500703.

Martínez G, Torres GA, Sarria G, Salcedo S, Varón F, Aya, H. 2008. Opciones de manejo de la Pudrición del cogollo (PC) de la palma de aceite en áreas de baja incidencia de la enfermedad. Revista Palmas 29(3): 63-67.

Marzin J, Daviron B, Rafflegeau S. 2014. Agricultures familiales et autres formes d'agriculture. In : Agricultures familiales et mondes à venir. Montpellier: Quae, pp. 75-92.

Munevar F, Acosta A, Leon-Gomez P. 2001. Factores edáficos asociados con la Pudrición de Cogollo de la palma de aceite en Colombia. Palmas 22: 9-19.

Naranjo F. 2014. Plan de acción contra la PC. Revista Institucional Palma 25: 8-12.

Nieto LE, Gómez P, Lozano C. 1996. Identificación y reproducción del complejo pudrición de cogollo de la palma de aceite (Elaeis guineensis Jacq.). Revista Palmas 17(2): 63-67.

Oil World. 2015. 17 Oils \& Fats: Summary of World Supply \& Demand, by Commodity. Available from: http://www.oilworld.biz (last consult 15/02/2016).

Rafflegeau S. 2007. Comparaison de stratégies de création de palmeraies non industrielles au Cameroun. In: Modélisation Économique des exploitations agricoles. Paris, France: L'Harmattan, pp. 23-43.

Rafflegeau S, Michel-Dounias I, Tailliez B, Ndigui B, Papy F. 2010. Unexpected N and K Nutrition Diagnosis in Oil Palm Smallholdings Using References of High-Yielding Industrial Plantations. Agronomy for Sustainable Development 30(4): 777-787. DOI: 10.1051/agro/2010019.

Rafflegeau S, Rioualec AL, Winterhalter J, Burgos R, Dubos B, Noboa I, et al. 2015. Los desafíos del desarrollo para los palmicultores de Quinindé y La Concordia. Revista Institucional Palma 26: 30-34.

Ronquillo-Narváez MP. 2012. Etiología de la pudrición del cogollo de la palma aceitera (Elaeis guineensis Jacq.) en el Ecuador. Puerto Rico, Ecuador: Universidad de Puerto Rico.

Savary S, Teng P, Willocquet L, Nutter FJ. 2006. Quantification and modeling of crop losses: a review of purposes. Annual Review of Phytopathologie 44: 89-112. 
Velez D, Norena C, Sarria G, Torres G, Varon F, Martinez G. 2008. Evaluacion y cuantificacion de estructuras de Phytophtora palmivora, el responsable de la pudricion del cogollo (PC) de la palma de aceite. Fitopatologia Colombiana 32(2): 33.
Winterhalter J. 2013. Analyse des performances agronomiques des parcelles et perception de la filière par les acteurs (QuinindéLaConcordia). Montpellier: Montpellier Sup'Agro-Institut des régions chaudes, $66 \mathrm{p}$. Mémoire de fin d'études.

Citation de l'article : Baron V, Rafflegeau S, Dubos B, Flori A, Burgos R, Louise C. 2017. Exposition des plantations de palmier à huile au risque de la pourriture du cœur dans le bassin de Quevedo, Équateur. Cah. Agric. 26: 55002. 